\title{
Preface
}

\section{A Comprehensive View of Noninvasive Ventilation}

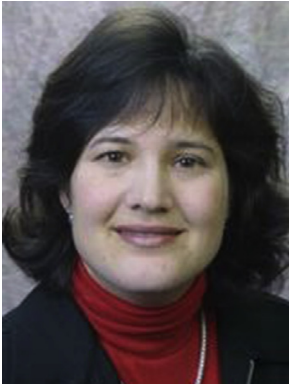

Lisa F. Wolfe, MD

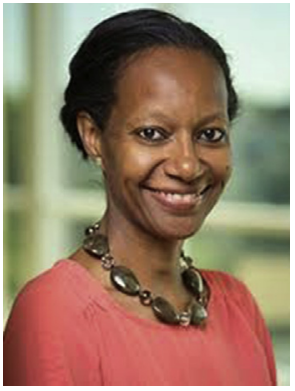

Amen Sergew, MD

Editors

Noninvasive ventilation (NIV), often referred to interchangeably as noninvasive positive pressure ventilation, is the focus of the current issue. One of the earliest uses of the modern day NIV machines dates to the 1940s when a group from Columbia University at Bellevue Hospital devised an "automatic respirator" to provide intermittent positive pressure ventilation using a facemask for patients with acute respiratory failure. ${ }^{1}$ NIV was used during the polio epidemic and was especially important for those with disabilities and chronic respiratory failure to be able to live independently in community-based living centers. ${ }^{2}$ However, in the decades that followed, invasive ventilation become the mainstay for the management of acute respiratory failure while NIV fell out of favor. During the 1980s and 1990s, NIV came back into focus as convincing evidence was published to support the use of NIV for the hospital-based treatment of acute exacerbations of chronic obstructive pulmonary disease (COPD) and acute pulmonary edema. ${ }^{3}$ NIV using a nasal mask (instead of a full facemask) was first reported in 1987 by Ellis and colleagues ${ }^{4}$ in 5 patients with neuromuscular weakness, which provided more data and options for the use of longterm home NIV.

Over the years, the indications for NIV have expanded to include various disease states. In this issue, the authors discuss the key disease states in which long-term NIV is used: obesity hypoventilation (Kaw and Kaminska), spinal cord injury (Daoud and colleagues), Duchenne muscular dystrophy (MacKintosh and colleagues), COPD (Orr and colleagues), amyotrophic lateral sclerosis (Cooksey and Sergew), Neuralgic Amyotrophy (Farr and colleagues), and common uses of NIV in the pediatric population (Shi and colleagues). Over time, various modes and settings have been developed to improve synchrony and portability. These devices and modes are discussed (Singh and $\mathrm{Cao}$ ) as well as the long-term follow up of NIV (Choi and colleagues). The use of NIV in the perioperative period (Dupuy-McCauley and Selim) and in the postacute phase, such as in long-term rehabilitation centers (Brown), is also discussed. NIV can be titrated in a sleep lab for optimal results when feasible and if outpatient titrations fail. Given the versatile patient population with vastly differing requirements, a thoughtfully designed and tailored sleep lab (Fiala and Coleman) provides more optimal and personalized results.

Our collection of articles provides a comprehensive review of the most pertinent issues in the use and management of NIV. We chose well-regarded experts in each topic, and we are immensely proud and grateful for their contributions. We are honored to have been a part of a series that will 
highlight NIV to educate our colleagues on this fast-moving literature and are thankful to Sleep Medicine Clinics for this opportunity.

Lisa F. Wolfe, MD Northwestern University Feinberg School of Medicine 676 North St. Clair Street, Suite 1400 Chicago, IL 60611, USA

Amen Sergew, MD National Jewish Health/University of Colorado 1400 Jackson St, B140 Denver, CO 80206, USA

E-mail addresses: Iwolfe@northwestern.edu (L.F. Wolfe) sergewa@njhealth.org (A. Sergew)

\section{REFERENCES}

1. Motley HL, Werko L, Cournand A, et al. Observations on the clinical use of intermittent positive pressure. J Aviat Med 1947;18(5):417-35.

2. Headley J. Edward Verne Roberts. In: PolioPlace. 2011. Available at: http://www.polioplace.org/ people/edward-verne-roberts. Accessed March 6, 2020.

3. Scala R, Pisani L. Noninvasive ventilation in acute respiratory failure: which recipe for success? Eur Respir Rev 2018;27(149).

4. Ellis ER, Bye PT, Bruderer JW, et al. Treatment of respiratory failure during sleep in patients with neuromuscular disease. Positive-pressure ventilation through a nose mask. Am Rev Respir Dis 1987; 135(1):148-52. 